\title{
Local anesthesia versus general anesthesia during endovascular therapy for acute stroke: a propensity score analysis
}

\author{
Raoul Pop (1) 1,2 François Severac, ${ }^{3}$ Emmanuel Happi Ngankou, ${ }^{4}$ Oana Harsan, ${ }^{2}$ \\ Ioan Martin, ${ }^{2}$ Dan Mihoc, ${ }^{2}$ Monica Manisor, ${ }^{2}$ Mihaela Simu, ${ }^{5}$ Salvatore Chibbaro, ${ }^{6}$ \\ Valérie Wolff, ${ }^{7}$ Roxana Gheoca, ${ }^{7}$ Alain Meyer, ${ }^{8}$ Julien Pottecher, ${ }^{8}$ Gérard Audibert, ${ }_{1}^{9,10}$ \\ Anne-Laure Derelle, ${ }^{4}$ Romain Tonnelet ${ }^{4}$ Liang Liao, ${ }^{4}$ François Zhu $0{ }^{4}{ }^{4}$ Serge Bracard, ${ }^{4}$ \\ René Anxionnat, ${ }^{4}$ Sébastien Richard, ${ }^{10,11,12}$ Rémy Beaujeux, ${ }^{1,2}$ Benjamin Gory ${ }^{4,10,13}$
}

\begin{abstract}
- Additional material is published online only. To view please visit the journal online (http://dx.doi.org/10.1136/ neurintsurg-2020-015916).
\end{abstract}

For numbered affiliations see end of article.

Correspondence to Dr Raoul Pop, Service de Neuroradiologie Interventionnelle, Hôpitaux Universitaires de Strasbourg, Strasbourg, France; pop.raoul@ gmail.com

Received 19 February 2020 Revised 24 March 2020 Accepted 2 April 2020 Published Online First 2 June 2020

\section{Linked}

- http://dx.doi.org/10.1136/ neurintsurg-2020-016512

Check for updates

(C) Author(s) (or their employer(s)) 2021. No commercial re-use. See rights and permissions. Published by BMJ.

To cite: Pop R, Severac F

Happi Ngankou E, et al.

J Neurolntervent Surg

2021:13:207-211.

\section{ABSTRACT}

Background To date, the choice of optimal anesthetic management during endovascular therapy (EVT) of acute ischemic stroke patients remains subject to debate. We aimed to compare functional outcomes and complication rates of EVT according to the first-line anesthetic management in two comprehensive stroke centers: local anesthesia (LA) versus general anesthesia (GA).

Methods Retrospective analysis of prospectively collected databases, identifying all consecutive EVT for strokes in the anterior circulation performed between January 1 , 2018 and December 31, 2018 in two EVT-capable stroke centers. One center performed EVT under LA in the first intention, while the other center systematically used GA. Using propensity score analysis, the two groups underwent 1:1 matching, then procedural metrics, complications, and clinical outcomes were compared. Good outcome was defined as 90 days modified Rankin Scale (mRS) $\leq 2$, and successful recanalization as modified Thrombolysis In Cerebral Ischemia (mTICl) 2b-3.

Results During the study period, 219 patients were treated in the LA center and 142 in the GA center. Using the propensity score, 97 patients from each center were matched 1:1 according to the baseline characteristics. Local anesthesia was associated with a significantly lower proportion of good outcome (36.1\% vs $52.0 \%$, OR 0.53 , $95 \% \mathrm{Cl} 0.33$ to $0.87 ; p=0.039$ ), lower rate of successful recanalization $(70.1 \%$ vs $95.8 \%$, OR $0.13,95 \%$ Cl 0.04 to $0.39 ; p<0.001)$, and more procedural complications (14.4\% vs $3.0 \%$, OR $3.44,95 \% \mathrm{Cl} 1.09$ to 14.28 ; $\mathrm{p}=0.018)$. There were no significant differences in 90-day mortality or symptomatic hemorrhagic transformation rates. Conclusions In this study, systematic use of GA for stroke EVT was associated with better clinical outcomes, higher recanalization rates, and fewer procedural complications compared with patients treated under LA as the primary anesthetic approach.

\section{BACKGROUND}

To date, the choice of optimal anesthetic management during endovascular therapy (EVT) of acute ischemic stroke patients remains subject to debate. ${ }^{1-3}$ Multiple retrospective studies and meta-analyses have associated the use of general anesthesia (GA) with lower odds of functional independence, when compared with patients treated without GA. ${ }^{12}$ In most of these studies there was no clear separation between patients treated under conscious sedation (CS) and local anesthesia (LA). These two groups were merged and analyzed together in a single 'nonGA' arm. Three recent randomized trials compared $\mathrm{CS}$ versus $\mathrm{GA}^{5-7}$ and failed to confirm inferiority of GA. On the contrary, a recent meta-analysis of these three randomized trials ${ }^{8}$ observed higher recanalization rates and better functional outcomes in patients treated under GA.

More recently, local anesthesia began to be studied separately from CS. Local anesthesia is defined as exclusive use of local anesthesia at the vascular access site, without sedation using inhaled or intravenous agents. A retrospective single-center study ${ }^{9}$ observed more favorable functional outcomes and decreased mortality in patients treated under LA compared with CS. Another recently published study comparing LA to $\mathrm{GA}^{10}$ found similar clinical outcomes. MR CLEAN Registry investigators recently published a retrospective analysis of outcomes depending on anesthesia type $^{3}$ and found that LA had a clear advantage over CS; however, this was less prominent compared with GA.

Overall, there are relatively few available data in the literature regarding the outcome of stroke thrombectomy under LA. All available studies have a significant limitation because of their design. Performing a retrospective selection of patients treated by different anesthesia methods within the same centers induces a strong selection bias - patients treated under LA may have a different and probably more benign clinical profile. These differences might not be entirely corrected for through post hoc multivariate analyses. Moreover, in real life, even when applying LA as the default anesthetic strategy, it is not possible to perform all endovascular procedures under LA. Some patients will not be suitable, because of agitation or instability, so they will need to be treated under CS or GA.

In order to address these issues, we aimed to assess the outcomes of stroke EVT under LA versus GA using a different approach. To reduce selection bias and evaluate 'real life' results when consistently applying each of the two anesthetic strategies, we 
compared outcomes for all consecutive patients with anterior circulation large-vessel occlusion treated with EVT in two comprehensive stroke centers situated in neighboring geographical regions.

\section{METHODS}

Data from this study are available from the corresponding author on reasonable request.

\section{PATIENT POPULATION}

We performed a retrospective analysis of prospectively collected endovascular procedure databases of stroke interventions performed between January 1, 2018 and December 31, 2018 in two neighboring comprehensive stroke centers with EVT on site. During this period, one center performed EVT under LA whenever possible. LA was defined as subcutaneous anesthesia at the arterial puncture site. In cases of excessive pain, intravenous injections of a light opioid analgesic (nalbuphine, $20 \mathrm{mg}$ bolus) and antiemetic (ondasetron $8 \mathrm{mg}$ bolus or metoclopramide $10 \mathrm{mg}$ bolus) were administered. CS was used only in cases of extreme agitation. GA was used only in the setting of depressed consciousness or in patients already intubated at the primary stroke center. In the second endovascular-capable center, EVT was systematically performed under GA for all patients. The anesthetic protocols for both centers are detailed in the online supplementary materials. In the present study, we included all patients presenting with acute ischemic stroke due to a large-vessel occlusion of the anterior circulation (internal carotid artery, M1 or M2 segment of middle cerebral artery, and tandem occlusions) in which an endovascular procedure was attempted. All consecutive cases were included, even in the setting of vascular access failures, EVT failure, or when EVT was not performed because the initial angiography revealed vessel recanalization (spontaneous or secondary to intravenous thrombolysis). There was no upper limit on age and admission National Institutes of Health Stroke Scale (NIHSS).

\section{OUTCOMES}

The primary outcome variable was the percentage of patients achieving good clinical outcome, defined as modified Rankin Scale (mRS) $\leq 2$ at 90 days. Secondary outcomes included successful recanalization, defined as modified Thrombolysis In Cerebral Ischemia $(\mathrm{mTICI})^{11} 2 \mathrm{~b}-3$, procedural complications, any intracerebral hemorrhagic (ICH) transformation, and 90-day mortality. Symptomatic ICH was defined as according to European Cooperative Acute Stroke Study (ECASS) 3 criteria. $^{12}$ Clinical status evaluation at 90 days was performed by the team of the first admittance center, either in person or by telephone, by a neurologist or a trained specialist nurse, certified in mRS evaluation. Clinical status assessors were independent and not involved in this study, but they were not blinded to other clinical details or anesthesia method.

\section{STATISTICAL ANALYSIS}

Continuous variables are expressed as median with IQR and were compared using Wilcoxon rank sum test. An assessment of the normality of the distribution was realized graphically and with Shapiro-Wilk test. Categorical variables are presented as numbers with percentages and were compared using Pearson $\chi^{2}$ test or Fisher exact test depending on theoretical numbers. In order to reduce the selection bias inherent in observational studies and to limit confusion bias, a propensity score matching analysis was performed. Individual propensity scores were calculated through multivariable logistic regression model accounting for clinically relevant determinants of clinical outcome and all variables significantly associated with the anesthetic strategy (local vs general anesthesia). After exclusion of subjects with a propensity score $>0.95$ or $<0.05$, patients with local or general anesthesia were matched with a ratio $1: 1$ on these propensity scores using a caliper size of 0.1 . The propensity score used to compare the two groups was calculated adjusting for all significant variables in univariate analysis, plus additional variables likely to influence clinical outcome. The criteria used for adjustment were as follows: age, baseline mRS, admission NIHSS and Alberta Stroke Program Early CT Score (ASPECTS) scores, time from symptoms onset to entry in angiography suite, prior intravenous thrombolysis, and vascular occlusion site at first angiographic run. The type of admission (direct to a EVT-capable center or secondary transfer) was not used for propensity score adjustment because it was significantly correlated with the time from symptoms onset to entry in angiography suite. After matching, categorical variables were compared using McNemar test or exact McNemar test depending on the theoretical numbers. When the test statistic was not computable, Bhapkar test was executed. Continuous variables were compared using Wilcoxon signed rank test. A P value $<0.05$ was considered as statistically significant. The matching was computed with the $\mathrm{R}$ MatchIt package and the analyses were realized using $\mathrm{R}$ software version 3.6.0 (R Core Team, 2019; R Foundation for Statistical Computing, Vienna, Austria; https://www.R-project.org/).

\section{RESULTS}

During the study period, 219 patients were treated in the LA center and 142 in the GA center. There were no significant differences in demographics and cardiovascular risk factors distribution (table 1). A significant proportion (60.3\%) of patients treated in the LA center were secondary transfers from a primary stroke center, whereas almost all the patients treated in GA center were primarily admitted. This difference was also reflected in a significantly longer time from symptoms onset to arrival in angiography suite in the LA center (72 min longer than the GA center). Admission NIHSS score was significantly higher in the LA center and intravenous thrombolysis was administered more frequently prior to EVT. Most patients in the LA center were treated under LA (81.7\%), the remaining were treated either under CS (16.4\%) or GA (1.8\%). A conversion to a different anesthesia method was done in $1.3 \%$ of cases. In the LA center, $19 / 179(10.6 \%)$ of patients treated under LA received intravenous injection of opioid analgesics (nalbuphine) at the beginning of EVT. In the GA center, all endovascular procedures were performed under GA (online supplementary table 1).

\section{PROPENSITY SCORE MATCHING AND SCORE-ADJUSTED ANALYSIS}

Using the propensity score, 97 patients from each center were matched 1:1 according to baseline characteristics. After matching, all covariates were statistically similar between the two groups (table 2), except for the modality of admission. However, despite this difference in modality of admission, the two matched groups had similar times from symptoms onset to entry in angiography suite. Local anesthesia was associated with a significantly lower proportion of good outcomes $(36.1 \%$ vs $52.0 \%$, OR $0.53,95 \%$ CI 0.33 to $0.87 ; \mathrm{p}=0.039)$, lower rate of successful recanalization (70.1\% vs $95.8 \%$, OR $0.13,95 \%$ CI 0.04 to 0.39 ; $\mathrm{p}<0.001)$, and more procedural complications (14.4\% vs 3.0\%, OR 3.44, $95 \%$ CI 1.09 to $14.28 ; \mathrm{p}=0.018$ ) (table 3 and figure 1 ). There were no significant differences in 90-day mortality or symptomatic ICH rates between the two groups. The use of GA was associated with a median delay of $19 \mathrm{~min}$ from entry in angiography suite to femoral 
Table 1 Patients' baseline characteristics

\begin{tabular}{|c|c|c|c|}
\hline Characteristic & $\begin{array}{l}\text { Local anesthesia } \\
\text { center }(n=219)\end{array}$ & $\begin{array}{l}\text { General anesthesia } \\
\text { center }(n=142)\end{array}$ & $P$ value \\
\hline Age (years) (median (IQR)) & $73(62-83)$ & $74(64-83)$ & 0.797 \\
\hline Male sex (n (\%)) & $108(49.3 \%)$ & $61(42.9 \%)$ & 0.221 \\
\hline \multicolumn{4}{|l|}{ Risk factors (n (\%)) } \\
\hline Hypertension & $139(63.4 \%)$ & $103(72.5 \%)$ & 0.093 \\
\hline Dyslipidemia & $71(32.4 \%)$ & $52(36.6 \%)$ & 0.446 \\
\hline Diabetes & $36(16.4 \%)$ & $34(23.9 \%)$ & 0.085 \\
\hline Smoking & $41(18.7 \%)$ & $16(11.2 \%)$ & 0.053 \\
\hline Baseline mRS score $>2$ & $17(7.7 \%)$ & $7(4.9 \%)$ & 0.276 \\
\hline \multicolumn{4}{|l|}{$\begin{array}{l}\text { Initial blood pressure }(\mathrm{mmHg}) \text { (median } \\
(\mathrm{IQR}) \text { ) }\end{array}$} \\
\hline Systolic & $147(131-165)$ & $146(130-165)$ & 0.92 \\
\hline Diastolic & $83(74-94)$ & $80(70-90)$ & 0.096 \\
\hline Left hemisphere stroke (n/N (\%)) & $110 / 213(51.6 \%)$ & $76 / 142(53.5 \%)$ & 0.877 \\
\hline \multicolumn{4}{|l|}{ Type of admission ( $\mathrm{n}(\%)$ ) } \\
\hline $\begin{array}{l}\text { Direct admission to comprehensive } \\
\text { stroke center }\end{array}$ & $87(39.7 \%)$ & $138(97.1 \%)$ & \\
\hline $\begin{array}{l}\text { Secondary transfer from primary } \\
\text { stroke center }\end{array}$ & $89(40.6 \%)$ & $4(2.8 \%)$ & $<0.001$ \\
\hline Other (including telemedicine) & $43(19.6 \%)$ & $0(0 \%)$ & 0.006 \\
\hline Baseline NIHSS (median (IQR)) & $16(12-20)$ & $14(8-20)$ & $<0.001$ \\
\hline $\begin{array}{l}\text { Symptom onset to entry in } \\
\text { angiography suite (min) (median (IQR)) }\end{array}$ & $270(198-379)$ & $198(150-294)$ & $<0.001$ \\
\hline $\begin{array}{l}\text { Symptom onset to femoral puncture } \\
\text { (min) (median (IQR)) }\end{array}$ & $295(226-411)$ & $248.5(191-340)$ & $<0.001$ \\
\hline IV thrombolysis (n (\%)) & $142(64.8 \%)$ & $51(35.9 \%)$ & 0.312 \\
\hline $\begin{array}{l}\text { Symptom onset to IV thrombolysis } \\
\text { (min) (median (IQR)) }\end{array}$ & $170(135-240)$ & $154(140-206)$ & \\
\hline \multicolumn{4}{|l|}{ Initial APECTS score } \\
\hline ASPECTS <5 (n/N (\%)) & $18 / 214(8.4 \%)$ & $18 / 140(12.8 \%)$ & 0.183 \\
\hline ASPECTS score (median (IQR)) & $8(6-8)$ & $8(6-9)$ & 0.046 \\
\hline \multicolumn{4}{|l|}{ Occlusion site at angiography (n (\%)) } \\
\hline MCA M1 segment & $115(52.5 \%)$ & $57(40.1 \%)$ & \\
\hline MCA M2 segment & $26(11.8 \%)$ & $36(25.3 \%)$ & \\
\hline Terminal ICA & $33(15.0 \%)$ & $25(17.6 \%)$ & \\
\hline Tandem occlusion & $27(12.3 \%)$ & $20(14.0 \%)$ & \\
\hline Extracranial ICA & $2(0.9 \%)$ & $0(0 \%)$ & 0.001 \\
\hline Bilateral & $1(0.04 \%)$ & $1(0.07 \%)$ & \\
\hline Recanalized & $15(6.8 \%)$ & $3(2.1 \%)$ & \\
\hline
\end{tabular}

ASPECTS, Alberta Stroke Program Early CT Score; ICA, internal carotid artery; IQR, interquartile range; IV, intravenous; MCA, middle cerebral artery; mRS, modifed Rankin Scale; NIHSS, National Institutes of Health Stroke Scale.

puncture (40 (31-50) vs 21 (16-28) min) (online supplementary table 2).

\section{DISCUSSION}

In the present study, we compared outcomes for acute ischemic stroke patients treated by EVT in two comprehensive stroke centers using two different first-line anesthetic strategies. Using a propensity score analysis, we found that the use of systematic GA compared with LA was associated with better 90-day clinical outcome, higher recanalization rates, and fewer procedural complications.

Our results are discordant with the findings of three previously published studies, which also compared outcomes of stroke EVT under LA versus GA. Two studies ${ }^{13} 14$ were in favor of LA; however, in both these studies, LA and CS were collectively referred to
Table 2 Patients' baseline characteristics after propensity score matching

\begin{tabular}{lccc}
\hline Characteristic & $\begin{array}{l}\text { Local anesthesia } \\
\text { center }(\mathbf{n}=97)\end{array}$ & $\begin{array}{l}\text { General anesthesia } \\
\text { center }(\mathbf{n}=97)\end{array}$ & P value \\
\hline Age (years) (median (IQR)) & $71(62-83)$ & $73(64-83)$ & 0.707 \\
\hline Male sex $(\mathrm{n}(\%))$ & $58 / 96(60.4 \%)$ & $45 / 97(46.3 \%)$ & 0.072 \\
\hline Risk factors $(\mathrm{n}(\%))$ & & \\
\hline Hypertension & $64(65.9 \%)$ & $73(75.2 \%)$ & 0.159 \\
\hline Dyslipidemia & $33(34.0 \%)$ & $38(39.1 \%)$ & 0.434 \\
\hline Diabetes & $17(17.5 \%)$ & $23(23.7 \%)$ & 0.288 \\
\hline Smoking & $17(17.5 \%)$ & $10(10.3 \%)$ & 0.144 \\
\hline Baseline mRS score $>2$ & $2(2.0 \%)$ & $5(5.1 \%)$ & 0.45
\end{tabular}

Initial blood pressure $(\mathrm{mmHg})$ (median

(IQR))

\begin{tabular}{|c|c|c|c|}
\hline Systolic & $145(128-162)$ & $150(140-170)$ & 0.241 \\
\hline Diastolic & $87(74-95)$ & $80(70-90)$ & 0.066 \\
\hline Left hemisphere stroke (n/N (\%)) & $52(53.6 \%)$ & $53(54.6 \%)$ & 0.598 \\
\hline \multicolumn{4}{|l|}{ Type of admission ( $\mathrm{n}(\%))$} \\
\hline $\begin{array}{l}\text { Direct admission to comprehensive } \\
\text { stroke center }\end{array}$ & $48(49.4 \%)$ & $94(96.9 \%)$ & \\
\hline $\begin{array}{l}\text { Secondary transfer from primary } \\
\text { stroke center }\end{array}$ & $35(36.0 \%)$ & $3(3.0 \%)$ & \\
\hline Other (including telemedicine) & $14(14.4 \%)$ & $0(0.0 \%)$ & $<0.001$ \\
\hline Baseline NIHSS (median (IQR)) & $15(11-18)$ & $16(8-21)$ & 0.898 \\
\hline $\begin{array}{l}\text { Symptom onset to entry in angiography } \\
\text { suite (min) (median (IQR)) }\end{array}$ & $265(180-335)$ & $190(150-314)$ & 0.109 \\
\hline $\begin{array}{l}\text { Symptom onset to femoral puncture } \\
\text { (min) (median (IQR)) }\end{array}$ & $284(192-355)$ & $253(188-371)$ & 0.645 \\
\hline IV thrombolysis (n (\%)) & $40(41.2 \%)$ & $39(40.2 \%)$ & 0.847 \\
\hline $\begin{array}{l}\text { Symptom onset to IV thrombolysis (min) } \\
\text { (median (IQR)) }\end{array}$ & $150(130-197)$ & $156(137-202)$ & 0.559 \\
\hline \multicolumn{4}{|l|}{ Initial APECTS score } \\
\hline ASPECTS $<5(n / N(\%))$ & $9(9.2 \%)$ & $12(12.3 \%)$ & 0.491 \\
\hline ASPECTS score (median (IQR)) & $8(6-9)$ & $8(7-9)$ & 0.396 \\
\hline \multicolumn{4}{|l|}{ Occlusion site at angiography (n (\%)) } \\
\hline MCA M1 segment & $40(41.2 \%)$ & $46(47.4 \%)$ & \\
\hline MCA M2 segment & $20(20.6 \%)$ & $15(15.4 \%)$ & \\
\hline Terminal ICA & $20(20.6 \%)$ & $22(22.6 \%)$ & \\
\hline Tandem occlusion & $15(15.4 \%)$ & $11(11.3 \%)$ & 0.704 \\
\hline Extracranial ICA & $0(0.0 \%)$ & $0(0.0 \%)$ & \\
\hline Bilateral & $0(0.0 \%)$ & $1(1.0 \%)$ & \\
\hline Recanalized & $2(2.0 \%)$ & $2(2.0 \%)$ & \\
\hline
\end{tabular}

ASPECTS, Alberta Stroke Program Early CT Score; ICA, internal carotid artery; IQR, interquartile range; IV, intravenous; MCA, middle cerebral artery; mRS, modified Rankin Scale; NIHSS, National Institutes of Health Stroke Scale.

as LA. A third and more recent publication, ${ }^{10}$ in which LA was correctly defined as an exclusive use of anesthesia at the arterial puncture site, observed similar outcomes between 'true' LA and GA. MR CLEAN Registry investigators published a retrospective analysis of outcomes depending on anesthesia type ${ }^{3}$ and found that LA had a clear advantage over CS; however, this was less prominent compared with GA. A sensitivity analysis was also performed, with groups based on center-level preference for LA, CS, or GA. However, both these studies were retrospective analyses of patients treated with different anesthesia methods within the same centers. We believe our results should be more representative for current clinical practice because we compared the outcomes of two firstline anesthetic strategies rather than two anesthetic methods. In current practice, even if LA is chosen as the default anesthetic 
Ischemic Stroke

Table 3 Type of anesthetic method and outcomes after propensity score matching

\begin{tabular}{|c|c|c|c|c|}
\hline Anesthetic method and outcomes & $\begin{array}{l}\text { Local anesthesia } \\
\text { center }(n=97)\end{array}$ & $\begin{array}{l}\text { General anesthesia } \\
\text { center }(n=97)\end{array}$ & $P$ value & OR $(95 \% \mathrm{Cl})$ \\
\hline \multicolumn{5}{|l|}{ Initial anesthesia method (n (\%)) } \\
\hline Local anesthesia & $76(78.3 \%)$ & $0(0.0 \%)$ & & \\
\hline Conscious sedation & $18(18.5 \%)$ & $0(0.0 \%)$ & N/A & $\mathrm{N} / \mathrm{A}$ \\
\hline General anesthesia & $3(3.0 \%)$ & $97(100 \%)$ & & \\
\hline Anesthesia conversion during procedure & $1(1.0 \%)$ & $0(0.0 \%)$ & & \\
\hline \multicolumn{5}{|l|}{ Angiographic outcome (n (\%)) } \\
\hline $\mathrm{mTICl} 2 \mathrm{~b}-3$ & $68(70.1 \%)$ & $93(95.8 \%)$ & $<0.001$ & 0.13 (0.04 to 0.39$)$ \\
\hline \multicolumn{5}{|l|}{ Procedural complications ( $\mathrm{n}(\%))$} \\
\hline Wire perforation & $3(3.0 \%)$ & $0(0.0 \%)$ & $<0.001$ & \\
\hline Embolization to new arterial territory* & $10(10.3 \%)$ & $1(1.0 \%)$ & 0.006 & \\
\hline latrogenic dissection & $2(2.0 \%)$ & $2(2.0 \%)$ & 1 & \\
\hline Totalt & $14(14.4 \%)$ & $3(3.0 \%)$ & 0.018 & 3.44 (1.09 to 14.28$)$ \\
\hline \multicolumn{5}{|l|}{ Imaging outcome ( $\mathrm{n} / \mathrm{N}(\%))$} \\
\hline Symptomatic ICH & $10 / 94(10.6 \%)$ & $7 / 93(7.5 \%)$ & 0.466 & $1.42(0.49$ to 4.34$)$ \\
\hline \multicolumn{5}{|l|}{ Clinical outcome at follow-up (n/N (\%)) } \\
\hline mRS score $\leq 2$ & $34 / 94(36.1 \%)$ & $50 / 96(52.0 \%)$ & 0.039 & $0.53(0.33$ to 0.87$)$ \\
\hline Mortality & $25 / 94(26.6 \%)$ & $21 / 96(21.8 \%)$ & 0.479 & 1.28 (0.60 to 2.77 ) \\
\hline \multicolumn{5}{|c|}{$\begin{array}{l}\text { Individual propensity scores were calculated accounting for baseline mRS score, time from symptoms onset to entry in angiography suite, admission NIHSS and ASPECTS scores, prior IV thrombolysis, and site of } \\
\text { occlusion at angiography. } \\
\text { *Persistent embolization in a new arterial territory (ENT) at the end of the procedure, excluding cases where ENT was successfully treated. } \\
\text { tOne patient had both perforation and ENT. } \\
\text { fFollow-up was unavailable in three cases for center A and one case for center B. } \\
\text { ASPECTS, Alberta Stroke Program Early CT Score; ICA, internal carotid artery; ICH, intracranial hemorrhage; IQR, interquartile range; IV, intravenous; MCA, middle cerebral artery; mRS, modified Rankin Scale; mTICI, } \\
\text { modified Thrombolysis In Cerebral Ischemia; NIHSS, National Institutes of Health Stroke Scale; OR, odds ratio. }\end{array}$} \\
\hline
\end{tabular}

method, it will be impossible to perform $100 \%$ of procedures under LA as some patients will not be suitable. Hence, the results in a group of patients with 100\% LA are less informative for realworld practice - in our study, a non-LA method was used in 19.2\% of cases in the center using first-line LA. Moreover, although our design was also retrospective, in comparison to previous studies, we have reduced patient selection bias by including all consecutive patients with EVT indication and treated in two different centers within the same recent time frame (year 2018).

The higher rate of patients who achieved functional independence in the systematic GA group is explained at least in part by the higher rate of successful recanalization achieved at the end of EVT, since recanalization status is a strong predictor of clinical outcome. ${ }^{15}$ Moreover, in our study, we also observed a significant higher rate of mTICI $2 c-3$ and mTICI 3 and lower rates of vascular
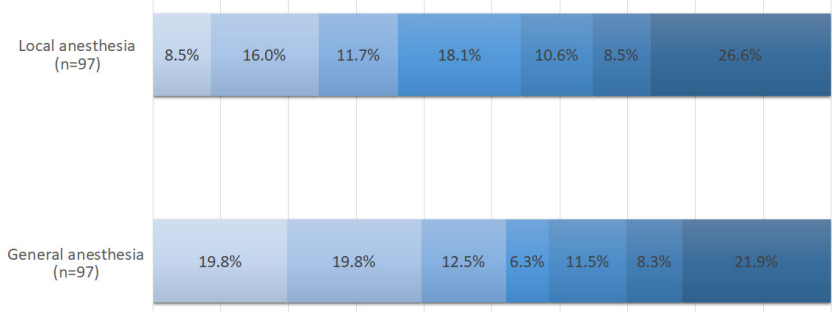

Figure 1 Distribution of modified Rankin Scale (mRS) scores at 90 days after propensity score matching. The mRS quantifies the degree of disability and dependence in daily activities. Patients with $\mathrm{mRS}$ scores $\leq 2$ are able to look after their own affairs without assistance. access failure under systematic GA. This result is in keeping with the findings in the recent meta-analysis ${ }^{16}$ of three randomized trials comparing GA with CS, also reporting a higher recanalization rate in the GA group. Taking these results into account, we can hypothesize that, in the setting of GA, operators are more likely to switch to alternative vascular access sites (brachial, radial, or direct carotid puncture) or continue the procedure and perform distal thrombectomy for residual emboli or emboli in a new territory (ENT), in the absence of patient agitation and discomfort. Another possible explanation for the difference in outcomes between the two groups is a significantly lower rate of procedural complications in the GA group. The rate of ENT was higher in the LA group and ENT has been associated with unfavorable prognosis. ${ }^{17}$ Moreover, we found significantly lower rates of arterial perforation in the GA group. This result is discordant with all previously published studies comparing either LA with $\mathrm{GA}^{10}$ or CS with GA, ${ }^{12}$ where there was no significant difference in perforation rates or global procedural complication rates.

One of the potential drawbacks of GA is a longer delay to treatment. Indeed, in our study the median time interval from arrival in the angiography suite to puncture was 19 min longer in the GA group. In the GOLIATH randomized trial, ${ }^{7}$ there was a median difference of $9 \mathrm{~min}$ between the CS group and GA group. In comparison to CS, LA does not induce any delay to treatment, because it does not require the presence of an anesthetist nor additional time to initiate sedation. Also, the longer delay in our study probably reflects 'real life' conditions, compared with the more standardized setting of a randomized trial; however, this difference should be used as a premise for improvement of in-hospital protocols in order to further reduce treatment delays.

Another potential drawback of GA is the occurrence of hypotension and increased blood pressure variability during induction and maintenance of anesthesia. In the meta-analysis of the three randomized studies comparing GA with $\mathrm{CS}^{8}{ }^{8}$ hypotension and 
blood pressure (BP) variability were significantly more common in the GA group. The interaction between periprocedural BP and functional outcomes is not fully understood. A recently published systematic review ${ }^{18}$ concluded that BP drops during thrombectomy seem to be associated with worse functional outcome, but periprocedural BP was not associated with functional outcome in studies with strict control of periprocedural BP. In our study we had a strict protocol for periprocedural BP control in the GA center but not in the LA center; however, we did not compare periprocedural BP levels between the two groups. We did not observe any differences between BP levels at admission. To date, no study has described periprocedural $\mathrm{BP}$ variations in patients treated under LA, so it is unknown whether correlations from CS versus GA analyses can apply in this context. Future research is needed to describe BP variations under LA and effects on functional outcome.

The main limitation of the present study is the fact that patients were retrospectively selected from two separate centers. Although all EVT procedures were performed by fully trained neurointerventionalists or by fellows in training under senior supervision, there might be inherent differences in technical approaches and experience levels between the two centers. Moreover, angiographic outcomes (mTICI scores) were not evaluated independently. Another limitation is the lack of data on periprocedural and postprocedural BP levels, which may impact clinical outcome.

\section{CONCLUSIONS}

Our study comparing outcomes of stroke thrombectomies in two centers using different anesthetic management protocols contradicts previous results, which showed worse outcomes for GA compared with non-GA. Although propensity score matching was used to eliminate baseline differences between the two groups, ideally a randomized clinical trial should be performed in order to provide a more definite conclusion.

\author{
Author affiliations \\ ${ }^{1}$ Interventional Radiology, Institut Hospitalo-Universitaire Strasbourg, Strasbourg, \\ France \\ ${ }^{2}$ Interventional Neuroradiology, University Hospitals Strasbourg, Strasbourg, Alsace, \\ France \\ ${ }^{3}$ Public Healthcare Department, University Hospitals Strasbourg, Strasbourg, Alsace, \\ France \\ ${ }^{4}$ Department of Diagnostic and Interventional Neuroradiology, University Hospital \\ Centre Nancy, Nancy, Lorraine, France \\ ${ }^{5}$ Neurology, University of Medicine and Pharmacy Victor Babes Timisoara, Timisoara, \\ Romania \\ ${ }^{6}$ Neurosurgery, University Hospitals Strasbourg, Strasbourg, Alsace, France \\ ${ }^{7}$ Stroke Unit, University Hospitals Strasbourg, Strasbourg, Alsace, France \\ ${ }^{8}$ Anesthesia, University Hospitals Strasbourg, Strasbourg, Alsace, France \\ ${ }^{9}$ Service d'Anesthésie-Réanimation Chirurgicale, Hôpital Central, University Hospital \\ Centre Nancy, Nancy, Lorraine, France \\ ${ }^{10}$ Faculte de Medecine, Universite de Lorraine, Nancy, Lorraine, France \\ ${ }^{11}$ Neurology Stroke Unit, University Hospital Centre Nancy, Nancy, Lorraine, France \\ ${ }^{12}$ U1116, INSERM, Nancy, France \\ ${ }^{13}$ U1254, INSERM, Nancy, France
}

\section{Twitter Raoul Pop @RaoulPop25}

Contributors RP, FS: acquisition, analysis and interpretation of data, drafting the manuscript, final approval of the version to be published, agreement to be accountable for all aspects of the study. OH, IM, DM, RG: acquisition of data, critical review of the manuscript, final approval of the version to be published, agreement to be accountable for all aspects of the study. EHN, MM, MS, SC, VW, AM, JP, GA, A-LD, $R T, L L, F Z, S B, R A, S R, R B$ : interpretation of data, critical review of the manuscript, final approval of the version to be published, agreement to be accountable for all aspects of the work. BG: conception and design of the study, analysis and interpretation of data, critical review of the manuscript, final approval of the version to be published, agreement to be accountable for all aspects of the study.

Funding The authors have not declared a specific grant for this research from any funding agency in the public, commercial or not-for-profit sectors.

Competing interests None declared.

\section{Patient consent for publication Not required.}

Ethics approval This is a retrospective report of procedures performed as part of usual patient care according to local institutional protocols. All presented data are anonymized.

Provenance and peer review Not commissioned; externally peer reviewed. Data availability statement Data are available upon reasonable request.

\section{ORCID iDs}

Raoul Pop http://orcid.org/0000-0003-4417-1496

François Zhu http://orcid.org/0000-0003-3838-9608

\section{REFERENCES}

1 Brinjikji W, Pasternak J, Murad MH, et al. Anesthesia-related outcomes for endovascular stroke revascularization: a systematic review and meta-analysis. Stroke 2017:48:2784-91.

2 Goyal N, Malhotra K, Ishfaq MF, et al. Current evidence for anesthesia management during endovascular stroke therapy: updated systematic review and meta-analysis. J Neurointerv Surg 2019;11:107-13.

3 Goldhoorn R-JB, Bernsen MLE, Hofmeijer J, et al. Anesthetic management during endovascular treatment of acute ischemic stroke in the MR CLEAN Registry. Neurology 2020;94:e97-106.

4 Campbell BCV, van Zwam WH, Goyal M, et al. Effect of general anaesthesia on functional outcome in patients with anterior circulation ischaemic stroke having endovascular thrombectomy versus standard care: a meta-analysis of individual patient data. Lancet Neurol 2018;17:47-53.

5 Schönenberger S, Uhlmann L, Hacke W, et al. Effect of conscious sedation vs general anesthesia on early neurological improvement among patients with ischemic stroke undergoing endovascular thrombectomy. JAMA 2016;316:1986-96.

6 Löwhagen Hendén P, Rentzos A, Karlsson J-E, et al. General anesthesia versus conscious sedation for endovascular treatment of acute ischemic stroke: the AnStroke trial (anesthesia during stroke). Stroke 2017;48:1601-7.

7 Simonsen CZ, Yoo AJ, Sørensen LH, et al. Effect of general anesthesia and conscious sedation during endovascular therapy on infarct growth and clinical outcomes in acute ischemic stroke. JAMA Neurol 2018;75:470-7.

8 Schönenberger S, Hendén PL, Simonsen CZ, et al. Association of general anesthesia vs procedural sedation with functional outcome among patients with acute ischemic stroke undergoing thrombectomy. JAMA 2019;322:1283-93.

9 van de Graaf RA, Samuels N, Mulder MJHL, et al. Conscious sedation or local anesthesia during endovascular treatment for acute ischemic stroke. Neurology 2018;91:e19-25.

10 Wu L, Jadhav AP, Zhao W, et al. General anesthesia vs local anesthesia during mechanical thrombectomy in acute ischemic stroke. J Neurol Sci 2019;403:13-18.

11 Zaidat 00, Yoo AJ, Khatri P, et al. Recommendations on angiographic revascularization grading standards for acute ischemic stroke. Stroke 2013;44:2650-63.

12 Hacke W, Kaste M, Bluhmki E, et al. Thrombolysis with alteplase 3 to 4.5 hours after acute ischemic stroke. N Engl J Med 2008;359:1317-29.

13 Abou-Chebl A, Zaidat 00, Castonguay AC, et al. North American SOLITAIRE Stent-Retriever Acute Stroke Registry: choice of anesthesia and outcomes. Stroke 2014:45:1396-401.

14 Abou-Chebl A, Yeatts SD, Yan B, et al. Impact of general anesthesia on safety and outcomes in the endovascular arm of Interventional Management of Stroke (IMS) III trial. Stroke 2015;46:2142-8.

15 Kaesmacher J, Dobrocky T, Heldner MR, et al. Systematic review and meta-analysis on outcome differences among patients with $\mathrm{TICI} 2 \mathrm{~b}$ versus $\mathrm{TICl} 3$ reperfusions: success revisited. J Neurol Neurosurg Psychiatry 2018;89:910-7.

16 Zhang Y, Jia L, Fang F, et al. General anesthesia versus conscious sedation for intracranial mechanical thrombectomy: a systematic review and meta-analysis of randomized clinical trials. J Am Heart Assoc 2019;8:e011754.

17 Chalumeau V, Blanc R, Redjem H, et al. Anterior cerebral artery embolism during thrombectomy increases disability and mortality. J Neurointerv Surg 2018;10:1057-62.

18 Maïer B, Fahed R, Khoury N, et al. Association of blood pressure during thrombectomy for acute ischemic stroke with functional outcome: a systematic review. Stroke 2019;50:2805-12. 